\title{
Retrospective analysis of surgery for cervical stump carcinoma at early stage
}

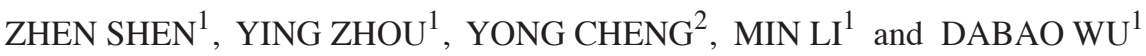 \\ Departments of ${ }^{1}$ Obstetrics and Gynecology, and ${ }^{2}$ Oncological Radiotherapy, \\ Anhui Provincial Hospital, Anhui Medical University, Hefei, Anhui 230000, P.R. China
}

Received February 12, 2017; Accepted November 21, 2017

DOI: $10.3892 / \operatorname{mco} .2017 .1517$

\begin{abstract}
Cervical stump cancer is a rare type of disease as total hysterectomies are performed infrequently. The purpose of this retrospective study was to assess the diagnosis, treatment, follow-up methods and complications of 10 patients with cervical stump carcinoma treated with surgery in Anhui Provincial Hospital affiliated to Anhui Medical University (Hefei, China). From January 2006 to October 2016 a total of 10 patients underwent surgery for carcinoma of the cervical stump. The pathological reports revealed $80 \%$ of cases were squamous cell carcinoma and $20 \%$ of cases were adenocarcinoma. The FIGO stage distribution was as follows: Carcinoma in situ (10\%); IB $_{1}(70 \%)$; IIA $(20 \%)$. The patients received a transvaginal trachelectomy or a radical trachelectomy and pelvic lymphadenectomy (either laparoscopic or laparotomic abdominal); four of the patients were treated with adjuvant chemotherapy, and two with concurrent chemoradiotherapy. The parametrial and resection margin infiltration, lymph node metastasis and lymph vascular space invasion (LVSI) were negative in all patients, and the deep stromal invasion rate was $66.7 \%$ (6/9). No incidences of recurrence or mortality were recorded during the follow-up interval of 6-120 months. Compared with the four patients who received laparotomic abdominal surgery, significantly less blood loss was recorded for the five patients who underwent laparoscopic surgery $(\mathrm{P}<0.01)$. There was no significant difference observed in the surgery time $(\mathrm{P}>0.01)$ or in the hospital stay duration $(\mathrm{P}>0.01)$ for the patients treated with laparotomic abdominal surgery and laparoscopic surgery. One patient experienced bladder fistula due to bladder over-dilation, but recovered quickly after the catheter was reinserted. Surgery for cervical stump cancer
\end{abstract}

Correspondence to: Dr Ying Zhou or Dr Dabao Wu, Department of Obstetrics and Gynecology, Anhui Provincial Hospital, Anhui Medical University, Hefei, Anhui 230000, P.R. China

E-mail: caddie1234@gmail.com

E-mail:dbwslyy@outlook.com

Key words: cervical stump cancer, laparoscope, laparotomic abdominal surgery at an early-stage is a viable and safer procedure, particularly the laparoscopic approach.

\section{Introduction}

Carcinoma of the cervical stump rarely occurs in patients who have previously received a subtotal hysterectomy, and accounts for $2-5 \%$ of all cervical cancer cases worldwide from 1970 (1-3). Barillot et al (1) analyzed 213 cases of cervical stump carcinoma and they observed that the locoregional control at equal time points between radiotherapy alone and surgery were very similar, however the lethal complications of radiotherapy were lower. Hellström et al (2) concluded that there was no difference in the longterm prognosis for radiologically treated squamous cell carcinoma cervical stump cancer and other cervical carcinomas, however the prognosis for adenocarcinoma stump cancers was worse than those in an intact uterus. For the treatment of early cervical stump cancer, Diaz-Feijoo et al (3) reported that total laparoscopic radical trachelectomy and intraoperative sentinel node identification was effective and safe. To date, there has been no standardized treatment of this type of cervical neoplasm. The majority of gynecological tumor experts conform to existing strategies of radiotherapy or laparotomic abdominal (or laparoscopic) radical surgery. Presentation $\geq 2$-years following a subtotal hysterectomy is considered to indicate true cervical stump cancer, whereas an interval of $<2$-years interval following subtotal hysterectomy is considered to denote a 'coincidental'carcinoma that may have been present at the time of the subtotal hysterectomy (4-7). In the present study, 10 cases of patients with cervical stump cancer were retrospectively analyzed with regard to the clinical characteristics (including age, clinical stage and histopathology), treatment and follow-up data. The results suggested that surgery for cervical stump cancer at an early-stage is a viable and safe procedure, particularly the laparoscopic approach as this type of surgery has fewer associated complications, including reduced blood loss and a shorter recovery time.

\section{Patients and methods}

Ethics statement. All the patients were recruited following the receipt of written consent between January 2006 and October 2016 at the Anhui Provincial Hospital affiliated to Anhui 
Medical University (Hefei, China). The study was reviewed and approved by the ethics review board of Anhui Provincial Hospital. All procedures performed in the study were in accordance with the ethical standards of the institutional research committee and with the 7th revised edition of the declaration of Helsinki.

Patient characteristics. A total of 10 patients were evaluated with respect to their medical history, a physical examination (including a pelvic examination), routine lab work and a chest $\mathrm{X}$-ray; the diagnosis was determined according to the system of the International Federation of Gynecology and Obstetrics (FIGO) by two or more gynecological tumor experts who were blinded to the patient data. Each patient had a histological diagnosis determined via biopsy prior to treatment.

The median patient age was $52.4 \pm 4.8$ years (range 46-61 years). All patients had previously undergone a subtotal hysterectomy for benign disease such as fibromyoma and menometrorrhagia. The mean time interval between the subtotal hysterectomy and diagnosis of cervical stump cancer was $11.4 \pm 5.6$ years (range, 5-21 years; all patients had an interval of $\geq 5$ years). The clinical data, including tumor stage and histology, are presented in Table I.

All the patients were followed up twice a year by gynecologic oncologists for ten years. A complete physical exam, including a pelvic examination, was performed on each visit. Vaginal smears and a colposcopy were conducted annually, as were routine chest X-rays and squamous cell carcinoma antigen studies. The median time of observation for patients alive at the last follow-up visit was $33.20 \pm 37.78$ months (range, 6-120 months).

Treatment. Patients with a cervical cancerous stump diagnosed as carcinoma in situ were administered a transvaginal trachelectomy. Patients with a cervical cancer stump diagnosed at stage $\mathrm{IB}_{1} / \mathrm{IIA}$ were treated with a radical trachelectomy and pelvic lymphadenectomy (8). A total of 5 cases were treated using a laparoscopic procedure: the laparoscope was placed into position $3 \mathrm{~cm}$ above the umbilicus through an 11- $\mathrm{mm}$ umbilical trocar with a direct-puncture, and three other trocars were introduced in the Trendelenburg position. Two accessory 12- and 5-mm trocars were then placed under direct visualization in the iliac fossae, and one 5-mm accessory trocar at the midline between the umbilicus and the left subcostal margin. The number and position of trocars were then used to perform a distal radical trachelectomy and a pelvic lymphadenectomy.

Following inspection of the abdominal cavity, the retroperitoneal spaces were opened and a systematic bilateral pelvic lymphadenectomy from the common iliac artery to the inferior boundary of the circumflex iliac vein was performed. Then, the paravesical, pararectal, rectovaginal, and vesicovaginal spaces were fully expanded, thereby delineating the uterosacral ligaments and the parametria. Uterine vessels were cut and coagulated at their origin from the hypogastric vessels; the parametrial tissue and the uterosacral ligaments were also transected by coagulation and cutting.

Dissection of tissue adhesion, the ureteral tunnel, urinary bladder and ureteral separation was performed with the aid of an Ultracision ${ }^{\circledR}$ Harmonic Scalpel (Ethicon, Inc., Cincinnati, $\mathrm{OH}, \mathrm{USA}$ ). The uterus-lifting cup was placed into the cervical
Table I. The clinical characteristics of the cervical stump cancer cases.

\begin{tabular}{lc}
\hline Variable & No. patients \\
\hline Age (years) & \\
$\leq 50$ & 4 \\
$>50$ & 6 \\
Clinical stage & \\
Carcinoma in situ & 1 \\
IB 1 & 7 \\
IIA & 2 \\
Histology & \\
Squamous carcinoma & \\
Keratin & 7 \\
Non-Keratin & 1 \\
Adenocarcinoma & 6 \\
High & 2 \\
Middle & 1 \\
Low & 1 \\
\hline
\end{tabular}

cavity to facilitate tissue dissection and the development of parametria and paracolpia. The proximal paracervical tissue and the vagina were transected and removed vaginally. The vaginal cuff was then closed laparoscopically by continuous suture. In total, four cases were treated with laparotomic abdominal radical trachelectomy and pelvic lymphadenectomy. A suprapubic catheter was inserted. Two of the patients subsequently received postoperative concurrent chemoradiotherapy due to deep stromal invasion. Four patients were treated with adjuvant chemotherapy (Paclitaxol and platinum).

\section{Results}

Histology. Squamous cell carcinoma was identified in $80 \%$ of cases, with the remaining $20 \%$ determined to be adenocarcinoma. This histological type distribution is similar that reported worldwide in the NCCN Guidelines ${ }^{\circledR} 2015$ in which squamous cell carcinoma accounted for $\sim 80 \%$ of all cervical cancer cases and adenocarcinoma accounted for $\sim 20 \%$ of all cases (8-12).

Age. The median age of patients with cervical stump carcinoma was 52.4 years, similar to the median age of 58 years reported by Miller et al (4), compared with 49 years in patients with cervical carcinoma of the intact uterus.

Clinical presentation. The clinical symptoms included vaginal bleeding (90\%) and abnormal cytology (10\%). No other symptoms were observed. All patients presented with hemoglobin levels of $>11 \mathrm{~g} / \mathrm{dl}$. The median interval between the onset of symptoms and a diagnosis was 5.45 months (Table II). In 10\% of cases, the symptoms were present for $\sim 2$ years prior to the establishment of a diagnosis. The mean operative time was $214.6 \mathrm{~min}$; the mean estimated blood loss was $180.0 \mathrm{ml}$ for laparoscopic surgery, and $687.5 \mathrm{ml}$ for laparotomic abdominal surgery (Fig. 1); the mean length of the hospital stay was 
Table II. The pathology patterns and the adjuvant treatment of patients with cervical stump cancer.

\begin{tabular}{ccccccccc}
\hline Case & $\begin{array}{c}\text { Diagnosis interval } \\
\text { (months) }\end{array}$ & $\begin{array}{c}\text { Differentiation } \\
\text { (Squamous or } \\
\text { adenocarcinoma) }\end{array}$ & $\begin{array}{c}\text { Deep stromal } \\
\text { invasion }\end{array}$ & $\begin{array}{c}\text { Parametrium and the } \\
\text { resection margin of the } \\
\text { vaginal involvement }\end{array}$ & $\begin{array}{c}\text { LN } \\
\text { Metastasis }\end{array}$ & LVSI CT & RT & $\begin{array}{c}\text { Follow-up } \\
\text { (months) }\end{array}$ \\
\hline 1 & 1 & Keratin & $<1 / 2$ & Neg. & Neg. & Neg. No No & 12 \\
2 & 2 & Non-Keratin & $>2 / 3$ & Neg. & Neg. & Neg. Yes No & 60 \\
3 & 4 & Non-Keratin & $>1 / 2$ & Neg. & Neg. & Neg. Yes Yes & 12 \\
4 & 24 & Non-Keratin & $>1 / 2$ & Neg. & Neg. & Neg. Yes No & 8 \\
5 & 6 & High & $>1 / 2$ & Neg. & Neg. & Neg. Yes Yes & 6 \\
6 & 3 & Non-Keratin & $<1 / 2$ & Neg. & Neg. & Neg. Yes No & 13 \\
7 & 0.5 & Middle & $>2 / 3$ & Neg. & Neg. & Neg. Yes No & 28 \\
8 & 2 & Non-Keratin & $<1 / 2$ & Neg. & Neg. & Neg. No No & 6 \\
9 & 6 & L & L & L & L & L & L L & 67 \\
10 & 6 & Non-Keratin & $>2 / 3$ & Neg. & Neg. & Neg. No No & 120 \\
\hline
\end{tabular}

LN, lymph node; LVSI, lymph vascular space invasion; CT, chemotherapy; RT, radiotherapy; L, data that was lost and cannot be collected; Neg, negative.

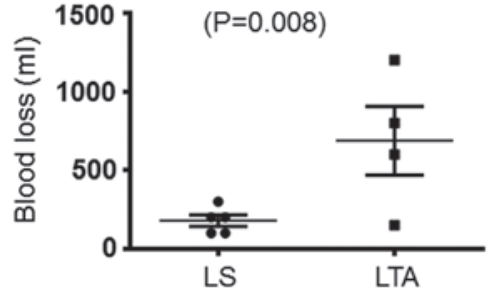

Figure 1. Scatter diagram of blood loss associated with LS and LTA $(\mathrm{P}<0.01)$. The mean estimated blood loss was $180.0 \mathrm{ml}$ for LS and $687.5 \mathrm{ml}$ for LAS. LS, laparoscopic surgery; LTA, laparotomic abdominal surgery.

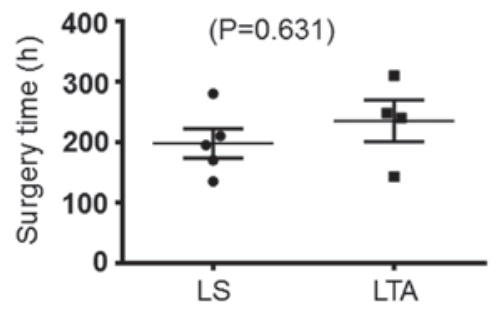

Figure 2. Scatter diagram of duration of the LS and the LTA $(\mathrm{P}>0.05)$. LS, laparoscopic surgery; LTA, laparotomic abdominal surgery.

14.8 days. The catheters of all patients were removed on the 21 st postoperative day after residual urine volumes of $80 \mathrm{ml}$. There was no significant difference in the surgery time and the duration of hospital stay between the laparoscopic surgery and the laparotomic abdominal surgery (Figs. 2 and 3). The pathological characteristics of the patients are presented in Table II. Overall, six patients were received adjuvant chemotherapy, two of whom also received combined radiotherapy (Table II).

Follow-up and complications. All the patients were followed-up and no cases of recurrence were observed; the mean follow-up time was 33.20 \pm 37.78 months (range, 6-120 months). No intraoperative or postoperative complications occurred, with the exception of one patient who experience bladder fistula that

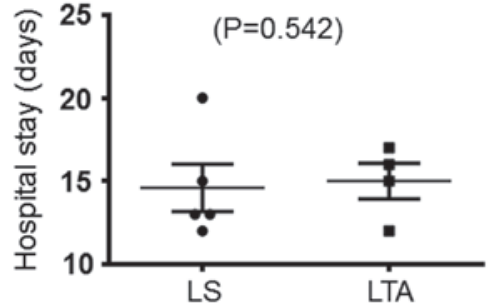

Figure 3. Scatter diagram of duration of hospital stay following LS and LTA $(\mathrm{P}>0.05)$. LS, laparoscopic surgery; LTA, laparotomic abdominal surgery.

occurred the day following the removal of the catheter due to bladder over-dilation; the patient recovered quickly after reinserting the catheter.

\section{Discussion}

Carcinoma of cervical stump accounts for 3-9\% of all cases of cervical cancer $(2,13)$. Miller et al (4) reported that the symptomatology, stage distribution and histology of cervical stump cancer were not significantly different to those of cervical carcinoma of the intact uterus. The individualization of therapy depends on the tumor stage, volume and depth of invasion. Although the 5-year survival rates of patients with cervical stump cancer compare favorably with patients with intact uteri, the complication rate appears to be enhanced by previous surgery and palliative care. Surgery is recommended for patients with pre-invasive disease and small volume $(2 \mathrm{~cm})$ invasive lesions limited to the cervix (stage IA and IB). In the present study, patients with stage IIA cervical stump cancer were surgically treated and no complications were identified.

A review of PubMed revealed that the majority of current reports are associated with the cornerstone management of radiation therapy; comparatively few reports are associated with radio-surgical combination methods. However, severe complications such as cystitis and rectum fistula have been reported, this is due to the absence of the uterus which acts as 
a shield. Of the present 9 cases treated with radical trachelectomy and pelvic lymphadenectomy (5 with laparoscopic; 4 with laparotomic abdominal surgery), only one patient experienced the complication of bladder fistula, and the patient recovered soon after reinserting the catheter.

Compared with patients with cervical cancer and intact uteri, the patients with cervical stump cancer were generally older and typically had concurrent complicating conditions such as hypertension or diabetes (14-19). The benefits of preserving the cervix may be associated with the patient's desire to preserve sexual, bladder and bowel function as previously reported, an approach that remains controversial among gynecologists (20-22). It must be noted that regular cervical screening following a subtotal hysterectomy is essential. Vaginal bleeding is a severe symptom in non-menstruating female.

As amputation of the radical cervical stump is considered to be challenging following a supravaginal hysterectomy, the majority of patients were subjected to concurrent chemoradiotherapy. Severe rectal, bladder and sigmoid complications following radiation have been reported due to the loss of protection offered by the intact uterus. Currently, surgery is the recommended approach for this type of tumor. With the development and proficiency of the laparoscopic surgical technique, ever more complicated laparoscopic surgical techniques have been made clinically available. This type of surgery has fewer associated complications, particularly with regard to reduced blood loss and a shorter recovery time.

In China, patients with malignant tumors often stay longer in hospital for reasons, including patient requests, age and physical weakness, compared with patients in developed countries. Oncologists must be vigilant to the appearance of complications. As the laparoscopic surgery approach has a number of inherent advantages, including easier identification of the ureter using laparoscopic enlargement and less injuries, laparoscopic surgery is recommended for the treatment of patients with early-stage cervical stump cancer.

\section{Acknowledgements}

The present study was supported by Anhui Provincial Science and Technology Agency 2013 Year Key Research Projects (grant no. 1301043053) and the National Natural Science Foundation of China (grant nos. 81001168 and 81272881). The Anhui Provincial Key Research and Development Project (grant no. 1704a0802151).

\section{References}

1. Barillot I, Horiot JC, Cuisenier J, Pigneux J, Schraub S, Rozan R, Pourquier H, Daly N, Vrousos C, Keiling R, et al: Carcinoma of the cervical stump: A review of 213 cases. Eur J Cancer 29A: 1231-1236, 1993.

2. Hellström AC, Sigurjonson T and Pettersson F: Carcinoma of the cervical stump. The radiumhemmet series 1959-1987. Treatment and prognosis. Acta Obstet Gynecol Scand 80: 152-157, 2001.
3. Diaz-Feijoo B, Gil-Moreno A, Puig O, Martínez-Palones JM and Xercavins J: Total laparoscopic radical trachelectomy with intraoperative sentinel node identification for early cervical stump cancer. J Minim Invasive Gynecol 12: 522-524, 2005.

4. Miller BE, Copeland LJ, Hamberger AD, Gershenson DM Saul PB, Herson J and Rutledge FN: Carcinoma of the cervical stump. Gynecol Oncol 18: 100-108, 1984.

5. Nass JM, Brady LW, Glassburn JR and Prasasvinichai S: The radiotherapeutic management of carcinoma of the cervical stump. Int J Radiat Oncol Biol Phys 4: 279-281, 1978.

6. Prempree T, Patanaphan V and Scott RM: Radiation management of carcinoma of the cervical stump. Cancer 43: 1262-1273, 1979.

7. Wolff JP, Lacour J, Chassagne D and Berend M: Cancer of the cervical stump. Study of 173 patients. Obstet Gynecol 39: 10-16, 1972.

8. Koh WJ, Greer BE, Abu-Rustum NR, Apte SM, Campos SM, Cho KR, Chu C, Cohn D, Crispens MA, Dorigo O, et al: Cervical Cancer, Version 2.2015. J Natl Compr Canc Netw 13: 395-404, 2015.

9. Johnson CJ, Graff R, Moran P, Cariou C and Bordeaux S: Breast cancer stage, surgery and survival statistics for Idaho's National Breast and Cervical Cancer Early Detection Program population, 2004-2012. Prev Chronic Dis 12: E36, 2015.

10. Rose J, Beaulac J, Howlett R and Kan L; Cervical Cancer Prevention and Control Network: Cervical cancer in Canada: A response to the release of the CCS/NCIC Cancer Statistics 2006. J Obstet Gynaecol Can 28: 678-679, 2006.

11. Bray F, Carstensen B, Møller H, Zappa M, Zakelj MP, Lawrence G, Hakama M and Weiderpass E: Incidence trends of adenocarcinoma of the cervix in 13 European countries. Cancer Epidemiol Biomarkers Prev 14: 2191-2199, 2005.

12. Bray F, Loos AH, McCarron P, Weiderpass E, Arbyn M, Møller H, Hakama M and Parkin DM: Trends in cervical squamous cell carcinoma incidence in 13 European countries: Changing risk and the effects of screening. Cancer Epidemiol Biomarkers Prev 14: 677-686, 2005.

13. Hannoun-Lévi JM, Peiffert D, Hoffstetter S, Luporsi E, Bey P and Pernot M: Carcinoma of the cervical stump: Retrospective analysis of 77 cases. Radiother Oncol 43: 147-153, 1997.

14. Maggi R, Bortolozzi G, Mangioni C, Uderzo A, Pisani G and Candiani GB: Residual cervical stump cancer (true cancer) and residual cancer of the cervical stump (coincident cancer). Casuistics and therapeutic results in the period 1955-1979. Eur J Gynaecol Oncol 6: 92-94, 1985.

15. Hellström AC, Hellman K, Pettersson BF and Andersson S: Carcinoma of the cervical stump: Fifty years of experience. Oncol Rep 25: 1651-1654, 2011.

16. Lee CL, Huang KG, Wang CJ, Yen CF and Soong YK: Radical laparoscopic surgery for carcinoma of the cervical stump. J Am Assoc Gynecol Laparosc 7: 241-244, 2000.

17. Liang Z, Xu H, Chen Y, Li Y, Chang Q and Shi C: Laparoscopic radical trachelectomy or parametrectomy and pelvic and para-aortic lymphadenectomy for cervical or vaginal stump carcinoma: Report of six cases. Int J Gynecol Cancer 16: 1713-1716, 2006.

18. Parkar RB, Hassan MA, Otieno D and Baraza R: Laparoscopic trachelectomy for cervical stump 'carcinoma in situ'. J Gynecol Endosc Surg 2: 58-60, 2011.

19. Shah AN and Olah KS: Cervical stump carcinoma following subtotal hysterectomy. J Obstet Gynaecol 22: 701, 2002.

20. Bey P, Dartois D, Schoumacher P and Pernot M: Carcinoma of cervical stump. A retrospective study (author's transl). Bull Cancer 67: 90-92, 1980.

21. Calais G, Leloup R, Chauvet B, Body G, Lansac J, Bougnoux P and Le Floch O: Carcinoma of the cervical stump. Retrospective analysis of 43 cases. Bull Cancer 76: 1103-1110, 1989 (In French).

22. Kovalic JJ, Grigsby PW, Perez CA and Lockett MA: Cervical stump carcinoma. Int J Radiat Oncol Biol Phys 20: 933-938, 1991. 